\title{
The Maverick Disease: Cystic Echinococcosis in Unusual Locations: A Ten Year Experience from an Endemic Region
}

\author{
Ayesha Butt ${ }^{1}$, Javaid Khan ${ }^{2}$ \\ 1. Medical College, Aga Khan University, Karachi, PAK 2. Internal Medicine: Pulmonology, Aga Khan University, \\ Karachi, PAK
}

Corresponding author: Ayesha Butt, ayeshabutt21@outlook.com

\section{Abstract \\ Background}

Cystic echinococcosis (CE) is a neglected tropical disease which affects more than 1 million people globally, causing a loss of 1-3 million disability-adjusted life years (DALYs) and a financial burden of US\$ 3 billion annually.The two most commonly involved organs are the liver and the lungs with involvement in $75 \%$ and 5 $-15 \%$ of cases respectively. The rest of the body can be involved in up to $10 \%$ of cases. In this study, we aim to explore the presentation, treatment and outcomes of $\mathrm{CE}$ in unusual locations.

\section{Methods}

Retrospective review of charts of 225 patients of CE admitted at Aga Khan Hospital, Karachi from 2007-2017 was done. Demographic information, date of admission, clinical presentation, laboratory and radiological findings, histopathology reports (where applicable), treatment course and outcomes were noted.

\section{Results}

CE occurred in the liver in 146 (64.9\%) patients, in the lungs in 55 (24.4\%) patients and in unusual locations in 24 (10.7\%) patients. Primary involvement of unusual locations was seen in 22 (91.7\%) cases. Amongst the 24 patients with disease in unusual locations, 13 (54.2\%) were males and 11 (45.8\%) were females and the median age of these patients was 43 years. Fever and dyspnea were the most common presenting complains, occurring in 5 (20.8\%) patients each followed by epigastric abdominal pain and weight loss occurring in $3(12.5 \%)$ patients each. Spleen was the most common unusual location for CE with four cases (16.7\%) of splenic involvement occurring, followed by cardiac, spinal and mediastinal involvement occurring in three (12.5\%) patients each. Other unusual locations included the abdominal cavity, bones, breast, kidney, seminal vesicle, brain, adrenal glands and the inguinal region. The treatment courses employed were a) medical, consisting of oral albendazole use( $400 \mathrm{mg}$ twice daily), b) surgical c) combined (medical plus surgical) therapy. Combined surgical and medical therapy, was the most common modality employed, with it being

Received 04/25/2019

Review began 04/29/2019 Review ended 10/11/2019 Published 10/18/2019

\section{() Copyright 2019}

Butt et al. This is an open access article distributed under the terms of the Creative Commons Attribution License CC-BY 3.0., which permits unrestricted use, distribution, and reproduction in any medium, provided the original author and source are credited. given to $14(58.3 \%)$ patients. Surgery only was performed in $5(20.8 \%)$ patients while medical therapy only to 3 (12.5\%) patients. Resolution of the disease was seen in 19 (79.2\%) patients on follow up imaging. Recurrence occurred in 4 (16.7\%) and mortality in 1 patient. Two patients (8.3\%) were lost to follow up.

\section{Conclusions}

CE can be challenging to diagnose especially when it occurs in unusual locations. CE must be included in the differential diagnosis of a cystic lesion in any organ of the body, especially in endemic areas, to ensure timely diagnosis and treatment, to prevent morbidity and mortality associated with chronicity of the disease.

Categories: Internal Medicine, General Surgery, Infectious Disease

Keywords: hydatid disease, neglected tropical diseases, parasitic diseases, zoonotic diseases, echinococcus granulosus infection

\section{Introduction}

Echinococcosis is a zoonotic parasitic disease caused by the larvae of dog and fox tapeworms of Echinococcus. Cystic echinococcosis (CE), also known as hydatid disease or hydatidosis, and alveolar echinococcosis (AE) are the two major types of the disease, caused by E. granulosus and E. multilocularis respectively. CE is much more common than AE and is classified as a neglected tropical disease (NTD). Although present globally, CE is highly endemic in the Mediterranean region, northern Africa, at the southern tip of South America, southern and eastern Europe, in Central Asia, western China and Siberia. More than 1 million people globally are afflicted by CE, causing a loss of 1-3 million disability-adjusted life years (DALYs) and a financial burden of US\$ 3 billion annually. Dogs serve as the definitive hosts and sheep, cattle, goats, and pigs as the intermediate hosts in the life cycle of E. granulosus. Dogs consume organs of 
other animals infested with hydatid cysts, which develop into adult tapeworms in dogs. Tapeworm eggs are then shed in feces of dogs and humans acquire the disease by ingestion of soil, water or food polluted with dog feces. Cystic echinococcosis was included by The World Health Organization (WHO) in a subdivision of selected NTDs to be tackled within its 2008-2015 strategic plan to control NTDs and WHO is now working towards establishing evidence based, impactful CE control strategies by 2020 [1].

Although CE can lead to formation of hydatid cysts in any part of the human body, the liver, followed by lungs are two organs classically involved in the disease [2]. However, in up to $10 \%$ of all cases, CE may occur in a kaleidoscope of unusual locations such as the spleen, heart, peritoneum, urinary tract, adrenal glands, ovary, brain, spinal cord, bone and soft tissues [3]. It is pertinent to study CE in unusual locations because CE diagnosis which is often missed even in the characteristic locations, becomes even more challenging when CE occurs in unusual locations. We aim to explore the unusual locations in which CE occurred in patients presenting to our center over a ten year period (2007-2017) and the presentation, treatment and outcomes of the disease in these patients.

\section{Materials And Methods}

The hospital records of 225 consecutive patients with a diagnosis of CE admitted at Aga Khan University Hospital, Karachi, from 2007-2017 were reviewed.

A diagnosis of CE had been made in the patients based on clinical history, laboratory and radiological findings and response to treatment, and confirmed by histopathological evaluation of samples in cases were surgical intervention was done. A diagnosis of CE depends on clinical manifestations, imaging and serology. The clinical criteria for diagnosis of CE requires presence of one of the following: slowly growing or static cystic mass(es) visible on imaging, anaphylaxis as a result of rupture or leakage of cysts or incidental finding of a cyst by imaging techniques. The diagnostic criteria comprises of: characteristic organ lesion(s) detected by radiological techniques, high-sensitivity serological tests confirmed by a separate high specificity serological test, histopathology or parasitology findings corroborating CE and pathognomonic gross appearance of cyst(s) in surgical specimens.

Demographic information, date of admission, clinical presentation, laboratory and radiological findings, histopathology reports (where applicable), treatment course and outcomes were noted in a questionnaire. For quantitative variable analysis IBM SPSS version 23 was used. Simple frequencies and proportions were calculated. The data was collected after attaining exemption from the Ethical Review Committee (ERC) of the Aga Khan University Hospital (ERC number 2019-1921-5281).

\section{Results}

A total of 225 patients with cystic echinococcosis were admitted in Aga Khan University Hospital over a ten year period. The patients included 126 males (56\%) and 99 females (44\%).There were 146 (64.9\%) cases of hepatic hydatid cysts, 55 (24.4\%) cases of pulmonary hydatid cysts and 24 (10.7\%) cases with unusual localizations of hydatid cysts.

Among the 24 patients with disease in unusual locations, 13 (54.2\%) were males and 11 (45.8\%) were females. The median age of these patients was 43 years (SD 13.9), with the greatest proportion of patients belonging to the $41-60$ years age group ( $\mathrm{n}=11,45.8 \%$ ) (Table 1$)$. 


\section{Cureus}

\begin{tabular}{|c|c|c|}
\hline CHARACTERISTIC & FREQUENCY & PERCENTAGE (\%) \\
\hline \multicolumn{3}{|l|}{ AGE GROUPS, YEARS } \\
\hline $0-20$ & 2 & 8.3 \\
\hline $21-40$ & 9 & 37.5 \\
\hline $41-60$ & 11 & 45.8 \\
\hline$>60$ & 2 & 8.3 \\
\hline \multicolumn{3}{|l|}{ SEX } \\
\hline Male & 13 & 54.2 \\
\hline Female & 11 & 45.8 \\
\hline \multicolumn{3}{|l|}{ EOSINOPHILIA } \\
\hline Absent & 22 & 91.7 \\
\hline Present & 2 & 8.3 \\
\hline \multicolumn{3}{|c|}{ ELISA FOR ANTI-ECHINOCOCCOSIS ANTIBODIES } \\
\hline Positive & 6 & 25 \\
\hline Negative & 3 & 12.5 \\
\hline Not done & 15 & 62.5 \\
\hline \multicolumn{3}{|c|}{ RADIOGRAPHIC INVESTIGATIONS AVAILABLE } \\
\hline Ultrasound & 6 & 25 \\
\hline Computerised Tomography(CT) Scan & 8 & 33.3 \\
\hline X-Ray & 1 & 4.2 \\
\hline Magnetic Resonance Imaging(MRI) & 2 & 8.3 \\
\hline \multicolumn{3}{|l|}{ IREAIMENI } \\
\hline Combined(Medical therapy+Surgery) & 14 & 58.3 \\
\hline Surgery only & 5 & 20.8 \\
\hline Medical therapy only & 3 & 12.5 \\
\hline OCCURRENCE OF COMPLICATIONS & 4 & 16.7 \\
\hline RECURRENCE & 4 & 16.7 \\
\hline LOST TO FOLLOW UP & 2 & 8.3 \\
\hline MORTALITY & 1 & 4.2 \\
\hline
\end{tabular}

TABLE 1: Descriptive characteristics of patients with CE in unusual locations: demographics, diagnostic modalities, treatment and outcomes $(n=24)$

Primary involvement of unusual locations was seen in 22 (91.7\%) cases. Only two patients had concomitant involvement of the liver in addition to CE in unusual locations. The unusual locations in which CE was seen is enlisted in Table 2. Fever and dyspnea were the most common presenting complains, occurring in 5 (20.8\%) patients each followed by epigastric abdominal pain and weight loss occurring in 3 (12.5\%) patients each. 


\section{Cureus}

\begin{tabular}{|l|l|l|}
\hline Location & Number of Cases & Percentage of total \\
\hline Spleen & 4 & 16.7 \\
\hline Heart & 3 & 12.5 \\
\hline Spine & 3 & 12.5 \\
\hline Mediastinum & 3 & 12.5 \\
\hline Abdominal cavity & 2 & 8.3 \\
\hline Kidney & 1 & 4.16 \\
\hline Kidney + Liver & 1 & 4.16 \\
\hline Left suboccipital region & 1 & 4.16 \\
\hline Left Tibia & 1 & 4.16 \\
\hline Right paracardiac +bilateral adrenal glands+left inguinal region. & 1 & 4.16 \\
\hline Right breast and chest wall & 1 & 4.16 \\
\hline Right Femur & 1 & 4.16 \\
\hline Right Hip Joint (Right proximal femur, acetabulum and greater sciatic notch) & 1 & 4.16 \\
\hline Seminal vesicle + Liver & 1 & 4.16 \\
\hline
\end{tabular}

TABLE 2: Unusual Locations of Cystic Echinococcus in the body $(n=24)$

Septic shock occurred in one patient. Two of the patients with splenic cysts presented with epigastric pain. The patient with a left suboccipital cyst presented with facial palsy, vomiting, vertigo and dizziness. Loss of appetite occurred in one patient with abdominal cysts while the other presented with melena. Dyspnea was the most common complain in patients with mediastinal cysts. Patients with cysts in the chest wall and the left tibia both presented with localized swelling. Moreover, whereas one patient with right hip involvement experienced localized pain, another one with proximal femur involvement presented with a discharging sinus. All patients with spinal involvement had lower limb weakness while one of them had urinary incontinence as well.

Upon workup, eosinophilia was present in only 2 (8.3\%) patients. The Echinococcus antibody Immunoglobulin $\mathrm{G}$ (IgG) assay which is an enzyme-linked immunosorbent assay (ELISA) based qualitative detection of IgG antibodies was used in 9 (37.5\%) patients. ELISA for anti-echinococcus antibodies was more than 1:16 in $6(25 \%)$ patients and less than 1:16 in $3(12.5 \%)$ patients (Table 1$)$. A CT scan was performed in 8 (33.3\%) patients while ultrasound was done in 6 (25\%). Imaging revealed a solitary cyst in 4 (16.7\%) patients.

The treatment courses employed were a) medical, consisting of oral albendazole use(400 mg twice daily), b) surgical c) combined (medical plus surgical) therapy. Combined surgical and medical therapy, was the most common modality employed, with it being given to $14(58.3 \%)$ patients. Surgery only was performed in 5 (20.8\%) patients while medical therapy only to 3 (12.5\%) patients.

Complications during treatment course occurred in 4 (16.7\%) patients. These complications included: Left ear discharge in the patient who underwent craniotomy for left suboccipital hydatid cysts, copious and clear wound discharge for which a lumbar drain was inserted in a patient with spinal CE and postoperative fever in two other patients.

Resolution of the disease was seen in 19 (79.2\%) patients on follow up imaging. Recurrence occurred in 4 (16.7\%) and mortality in 1 patient. Two patients (8.3\%) were lost to follow up.

\section{Discussion}

CE is grave public health issue afflicting populations spread across many different parts of the world and leading to significant morbidity and financial costs associated with treatment and the chronic nature of the disease [2]. The two most commonly involved organs are the liver and the lungs with involvement in $75 \%$ and $5-15 \%$ of cases respectively [3].The rest of the body can be involved in up to $10 \%$ of cases [4]. According to our findings as well, the liver emerged as the most common organ affected, comprising $64.9 \%$ of the total cases followed by pulmonary hydatid cysts in $24.4 \%$ of the cases whereas $10.7 \%$ cases had unusual 
localization.

Our results showed a greater percentage of male patients whereas previous studies from Pakistan have reported the male to female ratio in CE patients as 1:1.2 and 1:2.14 [5]. A systematic review of literature has also previously delineated CE to be more prevalent in females (Prevalence Proportion Ratio: 1.35 [95\% Bayesian Credible Interval: 1.16-1.53]) [6]. In contrast, in our study, the ratio of males to females was 1: 0.79 .

CE can cause cysts in any location in the body. A review delineated the most common unusual locations to be the central nervous system, musculoskeletal system, heart and kidney, whereas some less common locations included the spleen, pancreas, appendix, thyroid, salivary gland, adrenal gland, breast, and ovary [7].

CE afflicts the spleen in $0.5 \%$ to $11.3 \%$ of all CE cases and is considered to be the third most prevalent site of $\mathrm{CE}$ [2]. Hydatid cysts in the spleen can arise as a consequence of systemic spread due to rupture of a primary cyst at another location. Nonetheless, primary hydatid cysts can arise in the spleen, albeit very rarely [8-10]. In our study, cysts in the spleen were seen in $16.7 \%$ of cases, making it the most frequently involved unusual site of CE. All of these patients had isolated splenic involvement with hepatic and pulmonary involvement being excluded by imaging.

Osseous involvement by CE occurs in $0.5 \%$ to $2.5 \%$ of all CE patients and spinal involvement occurs in almost half of the cases [2]. The bone becomes primarily involved as a corollary of the settlement of bloodborne scolexes in bone. The proliferation of the cyst usually follows the bone canals and they may mimic locally malignant lesions on imaging. Hence, a final diagnosis can only be made by pathological assessment $[2,11]$. According to our results, six (25\%) patients had osseous CE, including patients with cysts in hip joint, femur, tibia and spine and half the cases involved the spine.

$\mathrm{CE}$ involves the central nervous system in $1 \%$ to $3 \%$ of patients and manifests as a space occupying lesion in the brain usually [2]. Intracranial hydatidosis is more frequently seen than spinal hydatidosis [12, 13]. The lesions are usually solitary and most often occur in the area supplied by the middle cerebral artery, particularly the parietal lobe. Brain hydatid cysts occur more frequently in children and are supratentorial [3, 14]. Amongst our patients, only one had a cerebral cyst, which was located in the subocciptal area.

CE in the urogenital system are rare. Renal CE is seen in only $1 \%$ to $4 \%$ of all CE cases [2]. Renal cysts may present a diagnostic challenge as they are difficult to differentiate from mass lesions and/or acquired cystic diseases of the kidney. In addition, these cysts are usually solitary, located at the upper pole or cortex and may reach sizes of up to $10 \mathrm{~cm}$ before producing any symptoms [2]. The route of infection of CE to the urinary tract is believed to be through the retroperitoneal lymphatics. A palpable mass, flank pain,malaise, hematuria, fever are common presentations while lower back pain is the most common symptom of renal CE [15]. The two patients in our study presented with lower back pain and decreased appetite respectively. Hydatid cysts in the seminal vesicles are extremely rare with scarce reports in literature [16-18]. Amongst our patients, only one had seminal vesicle involvement.

Hydatid cysts can develop in the heart in $0.2-2 \%$ of patients with CE [3, 14]. They may be a ramification of rupture of a lung hydatid cyst or may occur via spread by blood. Transthoracic echocardiography, computerized tomography (CT), and magnetic resonance imaging (MRI) can be employed for diagnosis. The most commonly affected cardiac areas are the left ventricle (50-60\% of cases), interventricular septum (10$20 \%$ ), the right ventricle (5-15\%), pericardium (10-15\%), and the right or left atrium (5-8\%) [3].

CE in the adrenal gland is also uncommon, and is usually a corollary of disseminated disease, as was the case in the one patient with adrenal involvement in our study, who had paracardiac, inguinal and adrenal cysts. The differential diagnosis of adrenal hydatid lesions includes endothelial cysts, pseudocysts, lymphangiomatous and angiomatous cysts and cystic degeneration of adrenal neoplasm [19].

Breast is an exceedingly rare location for CE to develop and accounts for only $0.27 \%$ of all cases [20, 21]. Mammography shows vague findings and the cyst usually appears as a circumscribed mass with thin peripheral calcification, similar to a simple cyst. On ultrasound, the lesion is usually indistinguishable from simple or complicated cysts [20,21]. On MRI, it appears as a well defined cystic lesion with capsular enhancement $[20,21]$. In our study, we encountered only one case of hydatid cyst in the breast and chest wall.

CE can be managed through four main modalities: medical therapy, percutaneous methods e.g. Puncture, Aspiration, Injection, Re-aspiration (PAIR) procedure, surgery and "wait and watch" [22]. Surgery is the treatment of choice for complicated, large or infected cysts, cysts at high risk of rupture and cysts in important organ locations [23]. Among our patients a combined management approach, consisting of surgery and albendazole was used in the majority $(58.3 \%)$ of patients.

Anti-parasitic medical therapy with benzimidazoles (BMZ), mainly albendazole, is effective for treating uncomplicated cysts, cysts in the liver or lungs in inoperable patients, cases with numerous cysts in two or 
more organs or peritoneal cysts. Moreover, pulmonary and hepatic CE1 and CE3a cysts less than $5 \mathrm{~cm}$ in diameter respond favorably to BMZ alone [23]. BMZ is also important in preventing recurrence following surgery or PAIR. However, BMZs alone should not be used against large cysts $(>10 \mathrm{~cm})$ and should be avoided in early pregnancy or against cysts likely to rupture [23]. A Watch and Wait approach is reserved for uncomplicated CE4 and CE5 cysts [23].

Serology has only a minor, confirmatory role due to a high rate of false negative results [23]. This is the reason why ELISA for anti-echinococcosis antibodies was done in only $37.5 \%$ of our patients. In the remaining patients, diagnosis was based on clinical presentation, radiological findings and histopathological evaluation of resected samples where applicable. Results of radiological investigation were accessible for only 17 out of 24 of our patients. The remaining patients had imaging reports from other center with them at time of presentation. The details of these imaging reports could not be accessed in our retrospective review conducted at our center, however, mention of imaging reports from other centers was found in charts of these 7 patients.

\section{Conclusions}

Cystic echinococcus can be challenging to diagnose especially when it occurs in unusual locations. Hence it is important to be cognizant that CE may be seen in any organ in the human body and must be included in the differential diagnosis of a cystic lesion in any organ especially in endemic regions to ensure timely diagnosis and treatment.

\section{Additional Information \\ Disclosures}

Human subjects: All authors have confirmed that this study did not involve human participants or tissue. Animal subjects: All authors have confirmed that this study did not involve animal subjects or tissue. Conflicts of interest: In compliance with the ICMJE uniform disclosure form, all authors declare the following: Payment/services info: All authors have declared that no financial support was received from any organization for the submitted work. Financial relationships: All authors have declared that they have no financial relationships at present or within the previous three years with any organizations that might have an interest in the submitted work. Other relationships: All authors have declared that there are no other relationships or activities that could appear to have influenced the submitted work.

\section{References}

1. Ahmed H, Ali S, Afzal MS, Khan AA, Raza H, Shah ZH, Simsek S: Why more research needs to be done on echinococcosis in Pakistan. Infect Dis Poverty. 2017, 6:90. 10.1186/s40249-017-0309-z

2. Gun E, Etit D, Buyuktalanci DO, Cakalagaoglu F: Unusual locations of hydatid disease: A 10-year experience from a tertiary reference center in Western Turkey. Ann Diagn Pathol. 2017, 29:37-40. 10.1016/j.anndiagpath.2017.01.011

3. Engin G, Acunas B, Rozanes I, Acunas G: Hydatid disease with unusual localization . Eur Radiol. 2000, 10:1904-1912. 10.1007/s003300000468

4. Siddappa S, Karthikeyan VS: Genitourinary hydatid disease: A review . Trop Med Surg. 2016, 4:208. 10.4172/2329-9088.1000208

5. Jamal Q, Jafarey NA: Hydatid disease at Jinnah Postgraduate Medical Centre Karachi . J Pak Med Assoc. 1989, 39:320-321.

6. Budke CM, Carabin H, Ndimubanzi PC, et al.: A systematic review of the literature on cystic echinococcosis frequency worldwide and its associated clinical manifestations. Am J Trop Med Hyg. 2013, 88:1011-1027. 10.4269/ajtmh.12-0692

7. Polat P, Kantarci M, Alper F, et al.: Hydatid disease from head to toe . Radiographics. 2003, 23:475-94. 10.1148/rg.232025704

8. Singal R, Sandhu KS, Mittal A, Gupta S, Jindal G: A giant splenic hydatid cyst . Proc (Bayl Univ Med Cent. 2016, 29:55-7. 10.1080/08998280.2016.11929359

9. Ozdogan M, Baykal A, Keskek M, Yorganc 1 K, Hamaloglu E, Sayek I: Hydatid cyst of the spleen: treatment options. Int Surg. 2001, 86:122 -6.

10. Kaya B, Uçtum Y, Kutanıș R: Splenic hydatid cyst attacking retroperitoneum. Turkiye Parazitol Derg. 2010, 34:193 -5. 10.5152/tpd.2010.12

11. Papanikolaou A: Osseous hydatid disease. Trans R Soc Trop Med Hyg. 2008, 102:233-238. 10.1016/j.trstmh.2007.09.012

12. Turgut M: Intracranial hydatidosis in Turkey: Its clinical presentation, diagnostic studies, surgical management, and outcome. A review of 276 cases. Neurosurg Rev. 2001, 24:200-208. $10.1007 / \mathrm{s} 101430100168$

13. Nourbakhsh A, Vannemreddy P, Minagar A, et al.: Hydatid disease of the central nervous system: A review of literature with an emphasis on Latin American countries. Neurol Res. 2010, 32:245-251. 10.1179/016164110x12644252260673

14. Keser SH, Selek A, Ece D, et al.: Review of hydatid cyst with focus on cases with unusual locations . Turk Patoloji Derg. 2017, 33:30 36. 10.5146/tjpath.2016.01369

15. Rexiati M, Mutalifu A, Azhati B, Wang W, Yang H, Sheyhedin I, Wang Y: Diagnosis and surgical treatment of renal hydatid disease: A retrospective analysis of 30 cases. PLoS One. 2014, 9:96602.

10.1371/journal.pone.0096602 


\section{Cureus}

16. Safioleas M, Stamatakos M, Zervas A, Agapitos E: Hydatid disease of the seminal vesicle: A rare presentation of hydatid cyst. Int Urol Nephrol. 2006, 38:287-289. 10.1007/s11255-006-6652-9

17. Papathanasiou A, Voulgaris S, Salpiggidis G, Charalabous S, Fatles G, Rombis V: Hydatid cyst of the seminal vesicle. Int J Urol. 2006, 13:308-310. 10.1111/j.1442-2042.2006.01288.X

18. Mushtaque M, Mir MF, Nazir P, Khan PS: Primary isolated echinococcal cyst of the seminal vesicle: A rare case. Urol Ann. 2012, 4:122-125. 10.4103/0974-7796.95571

19. Geramizadeh B, Maghbou M, Ziyaian B: Primary hydatid cyst of the adrenal gland: A case report and review of the literature. Iran Red Crescent Med J. 2011, 13:346-7.

20. Kumar A, Kumar A, Gaurav K, Chandra G, Tiwary AK, Bhagat S, Sarawgi M: A rare case of isolated hydatid cyst of breast. Int J Surg Case Rep. 2015, 7:115-118. 10.1016/j.ijscr.2014.10.093

21. Alamer A, Aldhilan A, Makanjuola D, Alkushi A: Preoperative diagnosis of hydatid cyst of the breast: A case report. Pan Afr Med J. 2013, 14:99. 10.11604/pamj.2013.14.99.2396

22. Brunetti E, Kern P, Vuitton DA: Expert consensus for the diagnosis and treatment of cystic and alveolar echinococcosis in humans. Acta Trop. 2010, 114:1-16. 10.1016/j.actatropica.2009.11.001

23. McManus DP, Gray DJ, Zhang W, Yang Y: Diagnosis, treatment, and management of echinococcosis. BMJ. 2012, 344:3866. 10.1136/bmj.e3866 